\title{
Remarks on Compact Automorphism Groups of a Certain von Neumann Algebra
}

\author{
By
}

\author{
Akitaka Kishiмото*
}

\begin{abstract}
Two remarks are given for a (faithful) continuous action of a compact group $G$ on a von Neumann algebra $M$. For a $G$-invariant normal state $\omega$ with central support 1 and with a one-sided $G$-spectrum, $M$ is shown to be isomorphic to the $\omega$-cyclic part of the fixed point subalgebra $M^{G}$ under some assumptions. A Galois correspondence is established between closed normal subgroups and von Neumann subalgebras of $M$ containing $M^{G}$ and globally invariant under $G$ and another subgroup $H$ of Aut $M$, which commutes with $G$ and acts ergodically on $M$.
\end{abstract}

\section{Introduction}

In a recent work [1] on extension of KMS states and chemical potential, Araki, Kastler, Takesaki and Haag carried out some structure analysis of a system consisting of a von Neumann algebra $M$ and a compact subgroup $G$ of Aut $M$ (the set of all *-automorphisms of $M$ ). The purpose of the present paper is to add two remarks to this analysis.

Our first result is about a $G$-invariant normal state $\omega$ of $M$ with the central support 1 which has a one-sided $G$-spectrum, in the sense that the set of continuous functions $\omega(x g(y)), x \in M, y \in M$, of $g \in G$ is orthogonal to either $p$ or its conjugate $\bar{p}$ for every $p \neq 1$ in the dual $\hat{G}$ of $G$. Under two further assumptions, we prove that $M$ is isomorphic to the $G$-fixed point subalgebra $M^{G}$ of $M$ restricted to the $\omega$-cyclic representation of $M^{G}$, one of the additional assumptions being the ex-

Communicated by H. Araki, October 12, 1976.

* Physics Department, Kyoto University, Kyoto, 606 Japan. 
istence of a subgroup $H$ of Aut $M$ which commutes with $G$, leaves $\omega$ invariant and acts ergodically on the center of $M$.

Our second result asserts the Galois correspondence between the set of closed normal subgroups of $G$ and the set of von Neumann subalgebras of $M$ containing $M^{G}$ and globally invariant under $G$ and $H$, where it is assumed that a subgroup $H$ of Aut $M$ commutes with $G$ and acts ergodically on $M$.

\section{One-sided Spectrum}

Let $\mathscr{H}$ be a Hilbert space and $M$ a $\sigma$-finite von Neumann algebra on $\mathscr{H}$. Let $G$ be a compact subgroup of Aut $M$. Suppose that $M$ has a cyclic vector $\Omega$ with $\|\Omega\|=1$ such that $(g(x) \Omega, \Omega)=(x \Omega, \Omega), x \in M$. Then there exists a (unique) continuous representation $U$ of $G$ such that $U(g) x \Omega=g(x) \Omega, x \in M, g \in G$. For $p \in \hat{G}$ let $\chi_{p}$ be the character of $p$ and

$$
\begin{aligned}
& E_{p}=\operatorname{dim} p \int \overline{\chi_{p}(g)} U(g) d g \\
& \varepsilon_{p}(x)=\operatorname{dim} p \int \overline{\chi_{p}(g)} g(x) d g, \quad x \in M,
\end{aligned}
$$

where $d g$ is the normalized Haar measure on $G$ and $\operatorname{dim} p$ is the dimension of the representation of class $p$. Then $E_{p}$ is a projection in the center of $U(G)^{\prime \prime}$ and

$$
\sum_{p \in G} E_{p}=1
$$

And $\varepsilon_{p}$ is a projection of $M$ onto $M(p) \equiv \varepsilon_{p}(M)$ and

$$
M=\left\{\bigcup_{p \in G} M(p)\right\}^{\prime \prime}
$$

Further we notice

$$
\mathscr{H}(p) \equiv E_{p} \mathscr{H}=[M(p) \Omega]
$$

where [] denotes the closed linear span. Let $S$ be the set $\{p \in \hat{G}$; $\mathscr{H}(p) \neq(0)\}$. Now we make 
Assumption $A . \quad S \cap \bar{S}=\{\ell\}$

where $\iota$ is the trivial representation of $G$.

Proposition 1. Under the above situation including Assumption A the following hold:

(i) $U(G) \subset M$

(ii) $E_{p} \in M^{G} \cap\left(M^{G}\right)^{\prime}$

(iii) $E_{p} M E_{p}=\left\{M^{G} \cup U(G)\right\}^{\prime \prime} E_{p}$

(iv) $\left(M \cap M^{\prime}\right) E_{p}=\left(M^{G} \cap\left(M^{G}\right)^{\prime}\right) E_{p}$.

Proof. We extend the action of $G$ to *-automorphisms of $B(\mathscr{H})$ as

$$
g(x)=\operatorname{Ad} U(g) x \equiv U(g) x U(g)^{*}, \quad x \in B(\mathscr{H}),
$$

and define $\varepsilon_{p}(x)$ for any $x \in B(\mathscr{H})$ by (2.2). Now suppose $M^{\prime}(p) \equiv \varepsilon_{p}\left(M^{\prime}\right)$ $\neq(0)$ for $p \in \hat{G}$ and let $y \in M^{\prime}(p)$ be nonzero. Then $y \Omega \in \mathscr{H}(p)$ and $y^{*} \Omega$ $\in \mathscr{H}(\bar{p})$ both do not vanish since $\Omega$ is separating for $M^{\prime}$. Hence $p$ and $\bar{p}$ both belong to $S$, i.e. $p \in S \cap \bar{S}$. By Assumption A we obtain $p=\imath$. By (2.3) with $M$ replaced by $M^{\prime}$, this implies $M^{\prime} \subset U(G)^{\prime}$ and so (i) holds.

Next by (i) and $M^{G} \subset U(G)^{\prime}$ we have $U(G)^{\prime \prime} \subset M \cap\left(M^{G}\right)^{\prime}$. Hence

$$
U(G)^{\prime \prime} \cap U(G)^{\prime} \subset M \cap\left(M^{G}\right)^{\prime} \cap U(G)^{\prime}=M^{G} \cap\left(M^{G}\right)^{\prime}
$$

which implies (ii).

The inclusion $E_{p} M E_{p} \supset\left\{M^{G} \cup U(G)\right\}^{\prime \prime} E_{p}$ is obvious. Since $U(G)^{\prime \prime} E_{p}$ is of (finite) type $I$ factor and

$$
E_{p} M E_{p} \cap U(G)^{\prime} E_{p}=M^{G} E_{p}
$$

we know that $U(G)^{\prime \prime} E_{p}$ and $M^{G} E_{p}$ generate $E_{p} M E_{p}$.

Finally (iv) follows from (iii) by computation:

$$
\begin{aligned}
\left(M \cap M^{\prime}\right)_{E_{p}} & =M_{E_{p}} \cap M_{E_{p}}^{\prime}=M_{E_{p}} \cap U(G)_{E_{p}}^{\prime} \cap\left(M^{G}\right)_{E_{p}}^{\prime} \\
& =M_{E_{p}}^{G} \cap\left(M^{G}\right)_{E_{p}}^{\prime}=\left(M^{G} \cap\left(M^{G}\right)^{\prime}\right)_{E_{p}} .
\end{aligned}
$$

Q.E.D. 
Let $H$ be a subgroup of Aut $M$ such that each $h \in H$ commutes with $g \in G$ and satisfies $(h(x) \Omega, \Omega)=(x \Omega, \Omega), x \in M$. Then there is a unitary representation $V$ of $H$ satisfying $h=\operatorname{Ad} V(h)$ and $V(h) \Omega=\Omega$. Then $V(h)$, $h \in H$ and $U(g), g \in G$, commute. Now we make

Assumption B. $\quad M \cap M^{\prime} \cap V(H)^{\prime}=\boldsymbol{C} 1$.

Proposition 2. For $p \in S$ the central support of $E_{p}$ in $M$ is 1 under Assumptions $A$ and $B$.

Proof. The central support of $E_{p}$ is in the commutant of $V(H)$ since so is $E_{p}$ itself and hence the proposition follows from Assumption B.

Our final assumption is

Assumption C. $M_{E \text {, }}^{G}$ is of type III.

Proposition 3. Under Assumptions $A, B$ and $C, E_{p}$ is purely infinite for $p \in S$.

Proof. By Assumption C $M_{E \iota}^{\prime}=\left(M^{G}\right)_{E \text { c }}^{\prime}$ is purely infinite; so is $M_{E_{p}}^{\prime}$ by Proposition 2. This implies that $M_{E_{p}}$ is purely infinite.

By Propositions 2 and 3 and by Corollary 5 in $[2$, p. 299] we have

Theorem 4. Under Assumptions $A, B$ and $C, M$ is isomorphic to $M_{E}^{G}$.

\section{Galois Correspondence}

Let $M$ be a von Neumann algebra and $G$ a compact subgroup of Aut $M$. Let

$$
\operatorname{Aut}_{G}(M)=\{\alpha \in \text { Aut } M ; \alpha \circ g=g \circ \alpha, g \in G\}
$$

We recall here some definitions in [1]. $\mathscr{H}_{G}(M)$ denotes the collection of all G-invariant finite dimensional Hilbert spaces $\mathfrak{S}$ in $M$ and $\mathscr{R}(G)$ denotes the collection of equivalence classes of unitary representa- 
tions of $G$ obtained by restricting $G$ to all members of $\mathscr{H}_{G}(M)$.

Basic assumption for our main result (Theorem 7 below) is as follows:

Assumption 1. There is a subgroup $H$ of $\operatorname{Aut}_{G}(M)$ whose action on $M$ is ergodic, i.e. $M^{H}=C 1$.

In the course of the proof, it is convenient to consider first the case satisfying the following assumption, the result of which is used to prove the same conclusion for the case not satisfying this assumption.

Assumption 2. The fixed point algebra $M^{G}$ of $M$ under $G$ is properly infinite.

Proposition 5. Under Assumptions 1 and 2, any $p \in \hat{G}$ belongs to $\mathscr{R}(G)$.

Proof. See (b) in the proof of Theorem III.3.3 and Appendix C in [1].

Proposition 6. Suppose $\hat{G} \subset \mathscr{R}(G)$. Let $\mathscr{H}$ be a subset of $\mathscr{H}_{G}(M)$ such that each $p \in \hat{G}$ is the equivalence class of an irreducible representation of $G$ obtained by restricting $G$ to some $\mathfrak{H} \in \mathscr{H}$. Then $\mathscr{H}$ and $M^{G}$ generate $M$. In particular $\mathscr{H}_{G}(M)$ generates $M$.

Proof. Let $N$ be the von Neumann algebra generated by $\mathscr{H}$ and $M^{G}$. First we suppose that Assumption 2 is satisfied. Let $\mathfrak{H} \in \mathscr{H}, U$ $=G \mid \mathfrak{H}$ and $n$ the dimension of the representation $U$ of $G$. Let $F_{n}$ be the $n \times n$ matrix algebra over $\mathbb{C}$ and let

$$
N_{n}=N \otimes F_{n}, \quad M_{n}=M \otimes F_{n}
$$

so that $N_{n} \subset M_{n}$. Set

$$
M_{n}^{\gamma}(U)=\left\{x \in M_{n} ; \gamma_{g}(x)=x\{1 \otimes U(g)\}, g \in G\right\}
$$

where $\gamma_{g}=g \otimes \iota$ is a $*$-automorphism of $M_{n}$. Then $\gamma$ leaves $N_{n}$ invariant and $N_{n}^{\gamma}(U)=M_{n}^{\gamma}(U) \cap N_{n}$ clearly contains a unitary $V$ : For example set $V_{i j}=u_{j} v_{i}^{*}$ where $\left\{u_{i}\right\}$ is the complete orthonormal system in $\mathfrak{S}$ by which 
$U$ is defined and $\left\{v_{j}\right\}$ is a complete orthonormal system in $M^{G}$ whose existence is assured by Assumption 2. Denoting $N_{n}^{\gamma}(U)$ and $M_{n}^{\gamma}(U)$ for the identity representation $U=\iota$ by $N_{n}^{\gamma}$ and $M_{n}^{\gamma}$ respectively, we have $N_{n}^{\gamma}=M_{n}^{\gamma}$, hence $N_{n}^{\gamma}(U)=N_{n}^{\gamma} V, M_{n}^{\gamma} V=M_{n}^{\gamma}(U)$ and $N_{n}^{\gamma}(U)=M_{n}^{\gamma}(U)$. For $x \in M$ let

$$
\varepsilon_{U}^{i, j}(x)=n \int \overline{U_{j i}(g)} g(x) d g
$$

Then $\left[\varepsilon_{U}^{i, j}(x)\right] \in M_{n}^{\gamma}(U)=N_{n}^{\gamma}(U)$ and hence

$$
\varepsilon_{U}(x) \equiv \sum_{i} \varepsilon_{U}^{i, j}(x) \in N
$$

for any $x \in M$. Thus we obtain $\varepsilon_{U}(M)=\varepsilon_{U}(N)$. Hence $\varepsilon_{p}(N)=\varepsilon_{p}(M)$ for all $p \in \hat{G}$. Since $\varepsilon_{p}(M)$ generate $M$, we obtain the conclusion $N=M$.

If $M^{G}$ is not properly infinite, let $\tilde{M}=M \otimes B(R)$ with the infinite dimensional Hilbert space $R$, let $\tilde{g}=g \otimes \ell$ be the automorphism of $\tilde{M}$ for each $g \in G$, and let $\widetilde{\mathscr{H}}=\{\mathfrak{H} \otimes 1, \mathfrak{S} \in \mathscr{H}\}$. Then applying the first part of the proof we conclude that $N \otimes C 1$ and $C 1 \otimes B(R)$ generate $\tilde{M}$; hence $N=M$.

Theorem 7. Under Assumption 1 there is a one-to-one correspondence between (i) the set of von Neumann subalgebras $N$ of $M$, containing $M^{G}$, globally $G$ - and $H$-invariant, and (ii) the set of closed normal subgroups $K$ of $G$, the correspondence being given by

$$
\begin{aligned}
& K=G_{N} \equiv\{g \in G ; g(x)=x, x \in N\}, \\
& N=M^{K} \equiv\{x \in M ; g(x)=x, g \in K\} .
\end{aligned}
$$

Proof. First we suppose that Assumption 2 is satisfied. Then it is known from Theorem III.3.3 (i) in [1] that for any closed subgroup $K$ of $G$,

$$
G_{\left(M^{K}\right)}=K
$$

If $K$ is normal, $M^{K}$ is in (i) as easily shown. Hence we have only to show that the mapping $K \rightarrow M^{K}$ is surjective.

Let $N$ be as in (i) and let $K=G_{N}$. Then $K$ is a closed normal subgroup of $G$ and $N \subset M^{K}$. Let $\mathscr{G}=G / K$. Then $\mathscr{G}$ acts on $\mathscr{N} \equiv M^{K}$ 
faithfully and leaves $N$ invariant; the action of $\mathscr{G}$ on $N$ is also faithful, and the fixed point algebras of $\mathscr{N}$ and $N$ under $\mathscr{G}$ are equal to $M^{G}$. By Proposition 5 we have $\hat{\mathscr{G}} \subset \mathscr{R}(\mathscr{G} \mid N)$. Hence Proposition 6 implies that $\mathscr{H}_{\mathscr{G}}(N)$ (as a subset of $\mathscr{H}_{\mathscr{G}}(\mathscr{N})$ ) and $M^{\mathbf{G}}$ generate $\mathscr{N}^{\prime}$, which forces $N=\mathscr{N}$. This completes the proot under Assumption 2.

In general case let $R$ be the infinite dimensional Hilbert space and let $\tilde{M}$ be as in the proof of Proposition $6, \widetilde{G}=\{g \otimes \iota ; g \in G\}$ and

$$
\widetilde{H}=\{h \otimes \sigma ; h \in H, \sigma \in \operatorname{Aut} B(\mathscr{R})\} .
$$

Then $\tilde{H} \subset \operatorname{Aut}_{\tilde{G}}(\tilde{M})$ and Assumptions 1 and 2 are satisfied for the system $(\tilde{M}, \widetilde{G})$. Now the first part of the proof and the following one-to-one correspondences complete the proof:

$$
\begin{aligned}
& K \longleftrightarrow \tilde{K}, \\
& M^{K} \longleftrightarrow \tilde{M}^{\tilde{K}}=M^{K} \otimes B(R),
\end{aligned}
$$

where $K$ are closed normal subgroups of $G$ and $\tilde{K}=\{k \otimes \imath ; k \in K\}$.

Remark. Our result (Theorem 7) is not yet satisfactory because we restrict our attention to only normal closed subgroups of $G$.

\section{Motivation}

In the following we state some of the results in [1] very briefly to explain the origin of our assumptions.

There is given a $C^{*}$-algebra $\mathscr{F}$ (algebra of fields), equipped with a continuous one-parameter group $\alpha$ of $*$-automorphisms (time evolution), a continuous representation $\gamma$ of a compact group $G$ by $*$-automorphisms (gauge group) and a $*$-automorphism $\tau$ (a space- or a time-translation). It is assumed that $\alpha_{t}(t \in \mathscr{R}), \gamma_{g}(g \in G)$ and $\tau$ mutually commute and that $\mathscr{F}$ is asymptotically abelian relative to $\tau$. Let $\mathfrak{A}$ be the $\gamma_{G}$-fixed point algebra $\mathscr{F}^{G}$ (the observable algebra) of $\mathscr{F}$, which is globally invariant under $\alpha_{t}(t \in \mathscr{R})$ and $\tau$. If a state $\omega$ of $\mathfrak{A}$ is weakly $\tau$-clustering, then an extension of $\omega$ to a weakly $\tau$-clustering state of $\mathscr{F}$ exists and is unique up to a translation by $\gamma_{G}[1$, Th. II.1]. Let $\phi$ be such an extension of $\omega$ and $G_{\phi}$ be the stabilizer of $\phi: G_{\phi}=\left\{g \in G ; \phi \circ \gamma_{\theta}=\phi\right\}$. If in addition $\omega$ 
satisfies the KMS condition relative to $\alpha_{t}$, then there are a continuous one-parameter subgroup $g_{t}$ in the normalizer of $G_{\phi}$ in $G$ and a closed normal subgroup $N_{\phi}$ of $G_{\phi}$ such that $\phi \mid \mathscr{F}^{N_{\phi}}$ satisfies the KMS condition relative to $\alpha_{t} \circ \gamma_{g_{t}}$ and the $N_{\phi^{-}}$-spectrum of $\phi$ is one-sided. Here the $N_{\phi^{-}}$ spectrum of $\phi$ is the set $S$ of all equivalence classes of irreducible unitary representations of $N_{\phi}$ which are contained in the unitary representation $U$ of $N_{\phi}$ in the GNS representation $\left(\mathscr{H}_{\phi}, \pi_{\phi}, \Omega_{\phi}\right)$ associated with $\phi$ satisfying

$$
U_{g} \pi_{\phi}(x) \Omega_{\phi}=\pi_{\phi}\left(\gamma_{g}(x)\right) \Omega_{\phi}, \quad x \in \mathscr{F}, g \in N_{\phi}
$$

And that the $N_{\phi}$-spectrum of $\phi$ is one-sided means that $p \in S$ is the trivial representation if the conjugate $\bar{p}$ also belongs to $S$.

Under the above situation we first notice

$$
\pi_{\phi}(\mathscr{F})^{\prime} \cap U_{\tau}^{\prime}=C \cdot 1
$$

where $U_{\tau}$ is defined as in (4.1) for the automorphism $\tau$. Let $\mathscr{N}=$ $\pi_{\phi}\left(\mathscr{F} N_{\phi}\right) "$ and let $E_{0}$ be the orthogonal projection onto $\left[\mathscr{N} \Omega_{\phi}\right]$. Then

$$
\mathscr{N}_{E_{0}} \cap\left(U_{\tau}\right)_{E_{0}}^{\prime}=C \cdot 1
$$

where of course $E_{0} \in \mathcal{N}^{\prime}$ and $E_{0} \in U_{\tau}^{\prime}$. Further

$$
\mathscr{N}_{E_{0}} \text { is of type III }
$$

except for trivial cases. (See the explanations above Theorem III. 3.3 in [1].)

Under this situation, $M, \Omega$ and $G$ in Section 2 are $\pi_{\phi}(\mathscr{F})^{\prime \prime}, \Omega_{\phi}$ and $N_{\phi}$ respectively. Assumption $\mathrm{A}$ is the one-sidedness of the $N_{\phi}$-spectrum of $\phi$ and Assumption B (resp. C) follows from (4.2) (resp. (4.4)).

$M, G$ and $H$ in Section 3 are $\pi_{\phi}\left(\mathscr{F}^{N_{\phi}}\right)^{\prime \prime}, G_{\phi} / N_{\phi}$ and $\left\{\bar{\tau}^{n}: n \in \mathbb{Z}\right\}$ respectively where $\bar{\tau}$ is the natural extension of $\tau$ to a *-automorphism of $\pi_{\phi}\left(\mathscr{F}^{N_{\phi}}\right)^{\prime \prime}$. Assumption 1 is due to (4.3) and Assumption 2 follows from (4.4).

Remark. In Section 2 if $M$ is a factor, Assumption B is trivially satisfied for the trivial group $H$. By Proposition 1 (iv) $M$ is a factor if $M_{E}^{G}$ is a factor; the latter assumption is reasonable in the above situa- 
tion.

\section{Acknowledgements}

The problems treated in this note were proposed to the author by Professor M. Takesaki during his seminar at RIMS, Kyoto University during the month of July, 1976. The author would like to thank Professor M. Takesaki for suggesting these problems and for discussions about them. He would also like to thank Professor H. Choda, Dr. Y. Nakagami and all other members of the seminar, with whom he enjoyed discussions.

\section{References}

[1] Araki, H., Kastler, D., Takesaki, M. and Haag, R., Extension of KMS states and chemical potential, Commun. Math. Phys., 53 (1977), 97-134.

[2] Dixmier, J., Les algèbres d'opérateurs dans l'espace hilbertien, Gauthier-Villars, Paris, 1957. 
